Annales Geophysicae (2001) 19: 17-23 C European Geophysical Society 2001

\title{
Statistical analysis of long-duration low-density solar wind events
}

\author{
S. Watari ${ }^{1}$, T. Watanabe ${ }^{2}$, and K. Marubashi ${ }^{1}$ \\ ${ }^{1}$ Communications Research Laboratory, 4-2-1 Nukuikita, Koganei, Tokyo 184-8795, Japan \\ ${ }^{2}$ Ibaraki University, 2-1-1 Bunkyo, Mito, Ibaraki 310-8512, Japan
}

Received: 3 July 2000 - Revised: 28 November 2000 - Accepted: 5 December 2000

\begin{abstract}
Low solar wind density with long duration was measured by in situ observation between 11 and 12 May 1999. As a result of this low-density solar wind condition, the magnetosphere of the Earth expanded considerably. We used a database of one-hour-averaged solar wind (19631999) near $1 \mathrm{AU}$ to determine whether or not the observed low-density event was extremely abnormal. As a result it was found that this event has the longest duration in approximately 36 years of solar wind observations.

There are three events with density $0.5 \mathrm{~cm}^{-3}$ or less and duration ten hours or longer. They were observed on 4 and 31 July 1979, and 11-12 May 1999. The 4 July 1979 event recurred on 31 July 1979 . The events were characterized by low-beta, low Alfven Mach number $\left(M_{A}\right)$, and low dynamic pressure. The occurrence rate of low-density solar wind with density $0.5 \mathrm{~cm}^{-3}$ or less shows several peaks near solar maxima. However, it is difficult to find a clear relationship between the sunspot number and the occurrence rate.
\end{abstract}

Key words. Interplanetary physics (flare and stream dynamics; solar wind plasma; sources of the solar wind)

\section{Introduction}

Long-duration low-density solar wind was observed between 11 and 12 May 1999. The balance between the magnetic pressure of the geomagnetic field and the dynamic pressure of the solar winds determines the size of the Earth's magnetosphere (Fairfield, 1971; Sibeck et al., 1991). The low-density solar winds cause a large expansion of the magnetosphere and the large outward excursion of the Earth's bow shock (Fairfield, 1971; Cairns et al., 1995; Takeuchi et al., 1998).

The solar wind conditions near $1 \mathrm{AU}$ have been measured since the early 1960s. However, this kind of long-duration low-density solar wind has not been fully studied. We studied historical low-density solar wind events using a large data set to better understand the event that occurred in May 1999.

Correspondence to: S. Watari (watari@crl.go.jp

\section{Data sources}

We used the one-hour-averaged solar wind parameters (density, speed, temperature, and magnetic field) collected between 1963 and 1999, compiled in the OMNI database. The solar wind data collected by the ACE spacecraft was used for the analysis of the events on 26-27 April 1999, 11-12 May 1999, and 28-30 June 1999.

Figure 1 shows the data coverage of the solar wind density observation for the OMNI database. Continuous solar wind observations by the WIND satellite, augmenting those of the IMP-8 satellite, have increased the coverage since 1995. An earlier period of good coverage was between 1973 and 1982 . In this period, the ISSE-3 spacecraft measured solar wind continuously at the L1 point. Between 1983 and 1994, the solar wind near $1 \mathrm{AU}$ was mainly measured using the IMP-8 satellite, and the averaged coverage was approximately $35 \%$.

Figure 2 shows the durations of low-density solar wind events (density $0.5 \mathrm{~cm}^{-3}$ or less). We consider solar wind density $0.5 \mathrm{~cm}^{-3}$ or less as "low-density" here. The May 1999 event is the longest low-density solar wind event studied. It lasted 22 hours. The second longest event occurred on 31 July 1979 and lasted 20 hours. The third longest event occurred on 4 July 1979 and lasted ten hours. The averaged duration of all low-density events was approximately four hours. Roughly half of the events lasted less than two hours.

Figure 3 shows yearly sunspot numbers and annual occurrence rates of low-density solar wind. No low-density events were observed between 1984 and 1996. One possible explanation for this is the low coverage of solar wind observations (see Fig. 1). However, low-density events were observed in 1966 in spite of approximately 35\% observation coverage. The occurrence rate shows several peaks near solar maxima (e.g., 1969, 1979, and 1999). Some events of low-density solar wind are associated with interplanetary transient disturbance events as discussed in the next section. Increase of the transient events near solar maxima might be related to these peaks. Figure 4 shows a scatter plot of yearly sunspot num- 


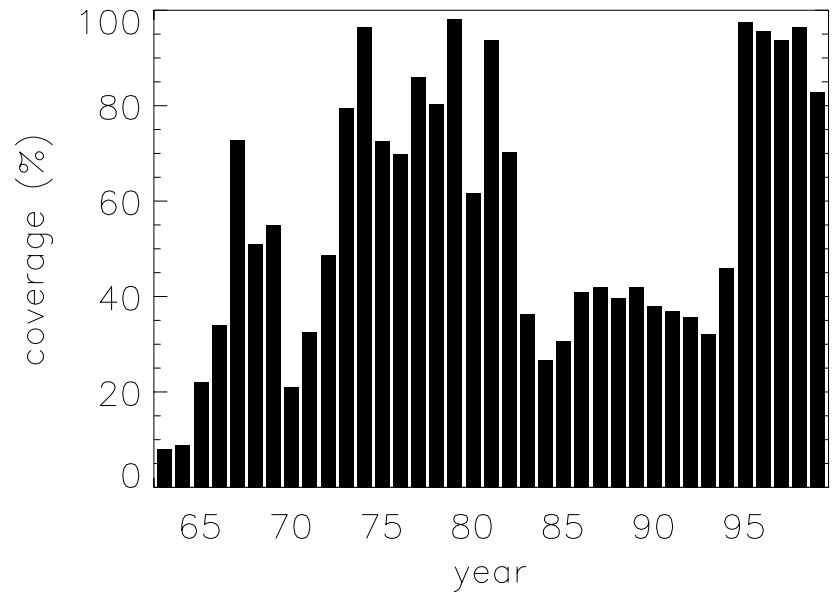

Fig. 1. Data coverage of solar wind density observations for OMNI data between 1963 and 1999.

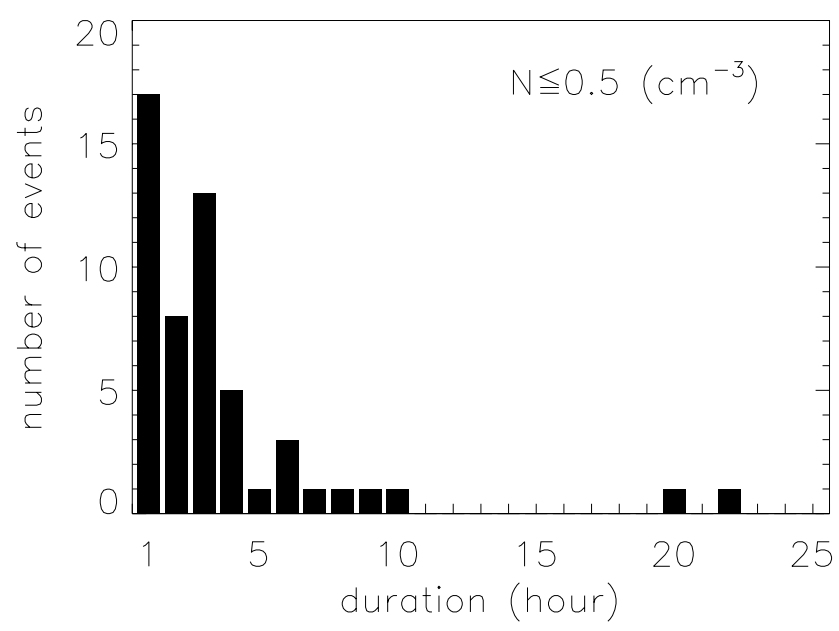

Fig. 2. A histogram of duration of low solar wind density events with density $0.5 \mathrm{~cm}^{-3}$ or less.

bers and annual occurrence rates of low-density solar wind. According to Fig. 4, it is difficult to find a clear relationship between sunspot numbers and occurrence rates of lowdensity solar wind.

Figure 5 shows scatter plots of density $(N)$ vs. speed $(V)$, $N$ vs. temperature $(T), N$ vs. magnetic field intensity $(|B|)$, $N$ vs. plasma beta $(\beta), N$ vs. dynamic pressure $\left(P_{\text {dyn }}\right)$, and square of speed $\left(V^{2}\right)$ vs. $P_{\text {dyn }}$. We calculated $P_{\text {dyn }}$ by the following equations: $P_{\mathrm{dyn}}=m_{p} N V^{2}$; where $m_{p}$ is the mass of proton. We used one-hour-averaged values between 1963 and 1999 to make Fig. 5, where the density varied between 0.1 and $100 \mathrm{~cm}^{-3}$, while the solar wind speed varied between 100 and $1000 \mathrm{~km} / \mathrm{s}$. The correlation coefficient between density and dynamic pressure, 0.73 is better than the coefficient between $V^{2}$ and $P_{\text {dyn }}, 0.23$. This suggests that the density is an important factor affecting the dynamic pressure of the Earth's magnetosphere. The correlation coefficients of $N$ vs. $V, N$ vs. $T, N$ vs. $|B|$, and $N$ vs. plasma beta are -0.35 , $-0.16,0.23$, and 0.08 , respectively.
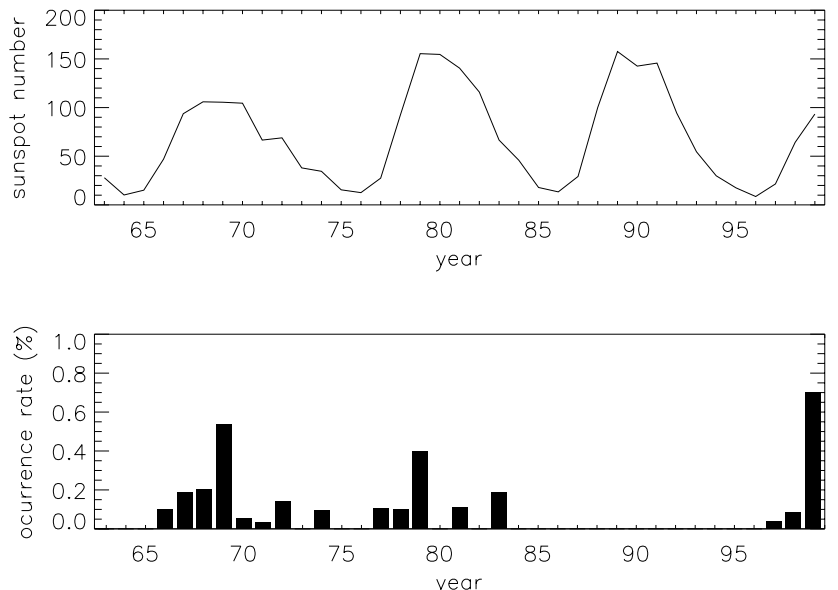

Fig. 3. Yearly sunspot numbers and annual occurrence rates of lowdensity solar wind (density $0.5 \mathrm{~cm}^{-3}$ or less).

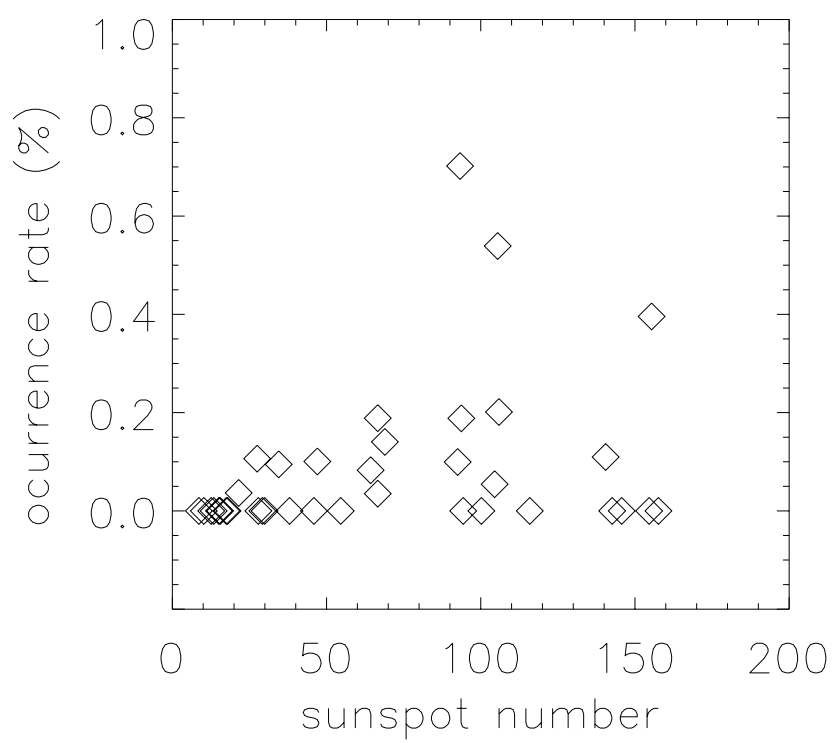

Fig. 4. A scatter plot of yearly sunspot numbers and annual occurrence rates of low-density solar wind (density $0.5 \mathrm{~cm}^{-3}$ or less).

Cairns et al. (1995) noted that Mach number is important for determining the shape and location of the Earth's bow shock and that solar wind with low Mach numbers causes large excursion of the Earth's bow shock. Figure 6 shows scatter plots of $N$ vs. Alfven Mach number $\left(M_{A}\right), V$ vs. $M_{A}$, $T$ vs. $M_{A}$, and $|B|$ vs. $M_{A}$. The correlation coefficients of $N$ vs. $M_{A}, V$ vs. $M_{A}, T$ vs. $M_{A}$, and $|B|$ vs. $M_{A}$ were 0.26 , $-0.04,-0.10$, and -0.50 , respectively. Smaller $M_{A}$ tends to be observed in lower solar wind density as shown in Fig. 6 .

\section{Historical events of low-density solar wind}

In Table 1 solar wind parameters of the events with solar wind density $0.5 \mathrm{~cm}^{-3}$ or less, and duration of three hours or longer, are summarized. According to Hundhausen (1997), 

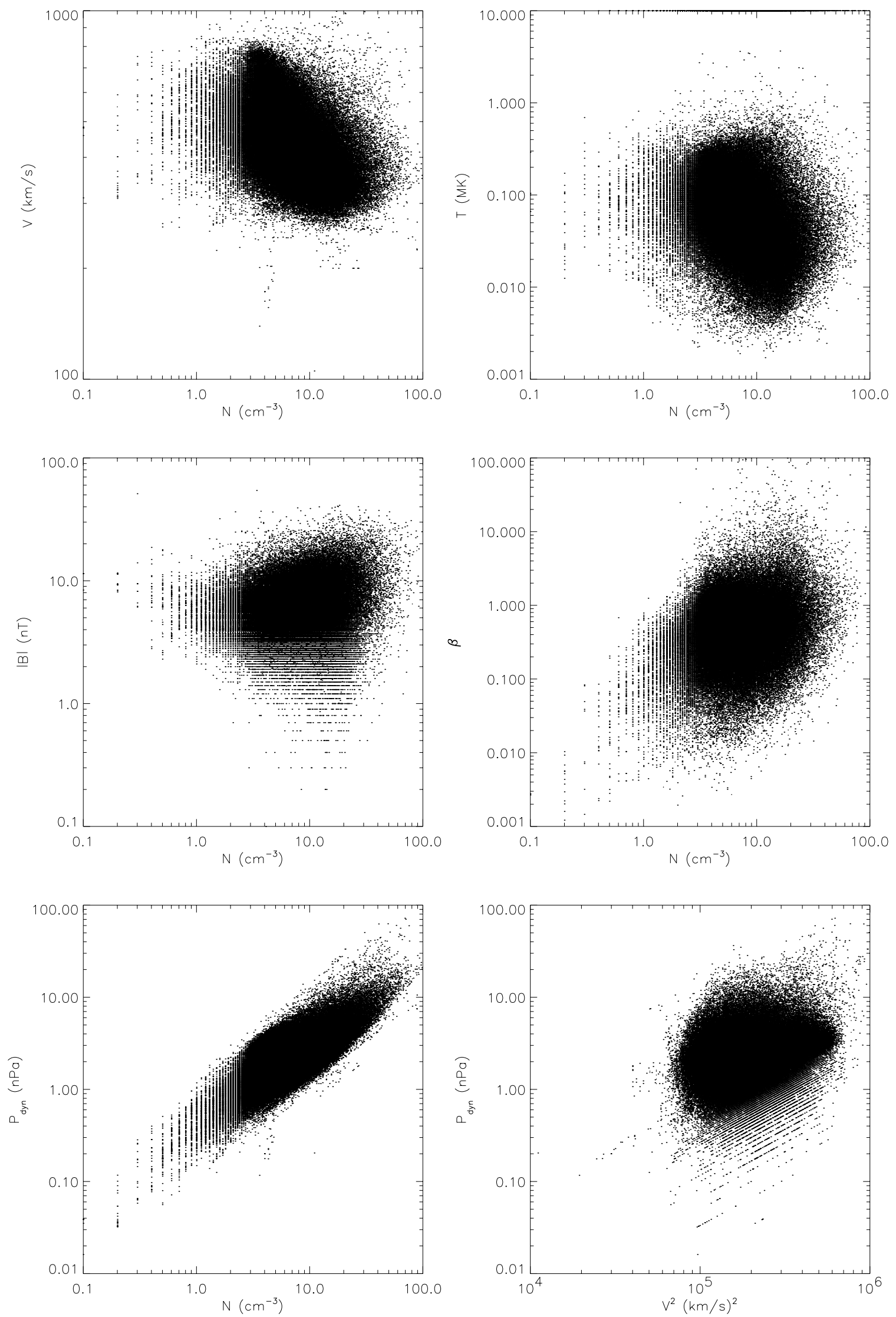

Fig. 5. Scatter plots of density $(N)$ vs. speed $(V), N$ vs. temperature $(T), N$ vs. magnetic field intensity $(|B|), N$ vs. plasma beta $(\beta), N$ vs. dynamic pressure $\left(P_{\mathrm{dyn}}\right)$, and square of speed $\left(V^{2}\right)$ vs. $P_{\mathrm{dyn}}$. 

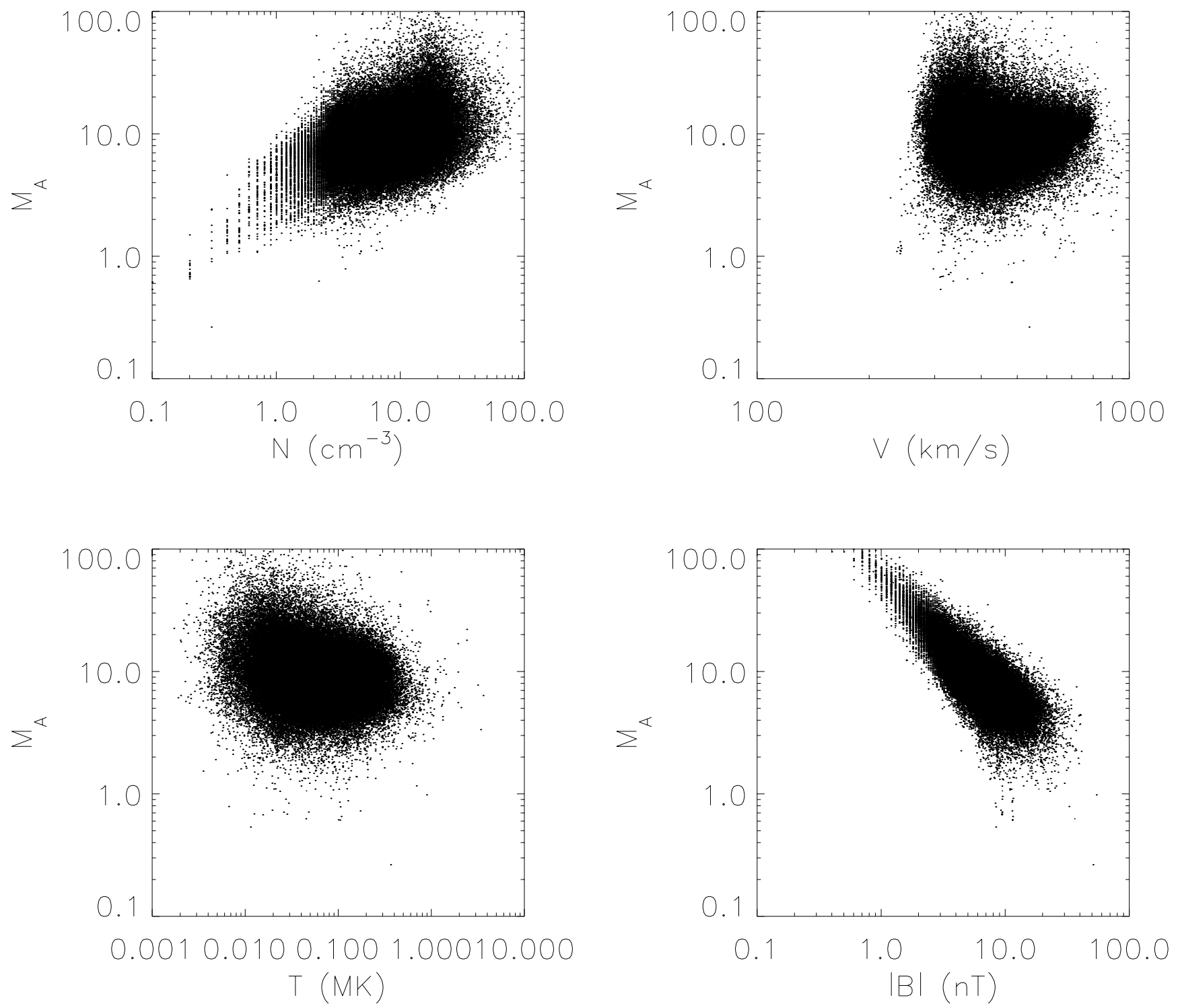

Fig. 6. Scatter plots of density $(N)$ and Alfven Mach number $\left(M_{A}\right)$, speed $(V)$ vs. $M_{A}$, temperature $(T)$ vs. $M_{A}$, and magnetic intensity $(|B|)$ vs. $M_{A}$.

typical values of density, speed, temperature, magnitude of magnetic field, beta, dynamic pressure, total pressure, and $M_{A}$ near $1 \mathrm{AU}$ are $6.6 \mathrm{~cm}^{-3}, 450 \mathrm{~km} / \mathrm{s}, 0.12 \mathrm{MK}, 7 \mathrm{nT}$, $0.56,2.23 \mathrm{nPa}, 0.049 \mathrm{nPa}$, and 7.57, respectively. Table 1 shows the dates and durations of the events and the averaged solar wind parameters (magnetic intensities, speeds, densities, temperatures, plasma beta, dynamic pressures, total pressures, and $M_{A}$ ). We calculated the total pressures ( $\left.P_{\text {tot }}\right)$ according to the following equations: $P_{\text {tot }}=P_{\text {gas }}+$ $P_{\mathrm{mag}}, P_{\mathrm{gas}}=N k\left(T_{p}+T_{e}\right), P_{\mathrm{mag}}=B^{2} / 2 \mu_{0}$; where $P_{\text {gas }}$, $P_{\mathrm{mag}}, k, T_{p}, T_{e}, B$, and $\mu_{0}$ are the gas pressure of solar wind, the magnetic pressure of solar wind, the Boltzmann constant, the proton temperature, the electron temperature, the magnetic field intensity, and the permeability of free space, respectively. We assumed that the electron density was equal to the proton density and that the $T_{e}$ was $141000 \mathrm{~K}$ and was constant (Newbury et al., 1998; Kawano et al, 2000). The low-density solar wind events in Table 1 are characterized by low values of beta, dynamic pressure, and $M_{A}$. Approximately $20 \%$ of the low-density events in Table 1 were associated with high-speed streams and approximately half of the events were associated with transient solar wind structures (e.g., flux rope, ejecta, and shock). The events associated with flux ropes were approximately $20 \%$ in Table 1 . High magnetic pressure inside flux ropes is a possible explanation for the low-density events. Several events (e.g., 1 and 27 December 1969, 4 and 31 July 1979) showed recurrent activities. The events on 1 and 27 December 1969 were observed in the tail of high-speed streams.

Figure 7 shows the solar wind parameters of three longduration, low-density solar wind events. The lines drawn at the level of $0.5 / \mathrm{cm}^{3}$ in the density panels serve to identify 
Table 1. Averaged values of solar wind parameters in the long-duration low-density solar winds

\begin{tabular}{|c|c|c|c|c|c|c|c|c|c|c|c|}
\hline no. & $\begin{array}{l}\text { Date } \\
\text { (UT) }\end{array}$ & $\begin{array}{l}\text { dur. } \\
\text { (hrs) }\end{array}$ & $\begin{array}{r}|B| \\
(\mathrm{nT})\end{array}$ & $\begin{array}{c}\text { speed } \\
(\mathrm{km} / \mathrm{s})\end{array}$ & $\begin{array}{l}\text { density } \\
\left(\mathrm{cm}^{-3}\right)\end{array}$ & $\begin{array}{l}\text { Temp. } \\
\text { (MK) }\end{array}$ & $\begin{array}{c}\text { plasma } \\
\text { beta }\end{array}$ & $\begin{array}{l}P_{\text {dyn }} \\
(\mathrm{nPa})\end{array}$ & $\begin{array}{c}P_{\text {tot }} \\
(\mathrm{nPa})\end{array}$ & $M_{A}$ & comments \\
\hline 1 & 1999/05/11 04 & 22 & 7.0 & 340 & 0.2 & 0.057 & 0.013 & 0.047 & 0.017 & 1.13 & \\
\hline 3 & 1979/07/04 09 & 10 & 9.1 & 453 & 0.2 & 0.126 & 0.005 & 0.061 & 0.052 & 0.79 & high-speed stream \\
\hline 4 & $1969 / 12 / 2718$ & 9 & 2.7 & 513 & 0.5 & & & 0.204 & & 5.98 & tail of high-speed stream \\
\hline 7 & 1972/03/10 06 & 6 & & 262 & 0.5 & 0.132 & & 0.057 & & & largely dropped speed? \\
\hline 8 & $1999 / 04 / 2700$ & 6 & 9.3 & 397 & 0.4 & 0.086 & 0.014 & 0.106 & 0.036 & 1.24 & \\
\hline$* 19$ & $1999 / 06 / 3020$ & 6 & 6.1 & 455 & 0.4 & 0.102 & 0.041 & 0.153 & 0.016 & 2.26 & after a fast transient event \\
\hline 10 & 1998/05/05 08 & 5 & & 570 & 0.4 & 0.063 & & 0.229 & & & associated with a fast ejecta \\
\hline 11 & 1968/02/09 07 & 4 & 6.2 & 514 & 0.5 & 0.097 & 0.042 & 0.209 & 0.017 & 2.62 & associated with ejecta \\
\hline 15 & $1999 / 06 / 2809$ & 4 & 4.9 & 742 & 0.4 & 0.185 & 0.110 & 0.353 & 0.011 & 4.29 & associated with a fast ejecta \\
\hline 16 & $1966 / 03 / 2209$ & 3 & & 473 & 0.5 & 0.018 & & 0.187 & & & data gap \\
\hline 17 & 1967/01/14 10 & 3 & 10.4 & 456 & 0.5 & 0.034 & 0.005 & 0.173 & 0.044 & 1.43 & followed a flux rope \\
\hline 18 & 1967/08/01 03 & 3 & 7.2 & 439 & 0.5 & 0.069 & 0.022 & 0.150 & 0.022 & 1.90 & data gap \\
\hline 19 & 1969/03/01 10 & 3 & & 534 & 0.5 & 0.153 & & 0.239 & & & after a fast ejecta \\
\hline 20 & $1969 / 03 / 2600$ & 3 & 9.0 & 605 & 0.5 & & & 0.306 & & 2.18 & associated with a fast ejecta \\
\hline$*^{*} 21$ & 1969/12/0103 & 3 & 4.3 & 522 & 0.5 & & & 0.227 & & 3.93 & tail of high-speed stream \\
\hline${ }^{* 4} 22$ & $1969 / 12 / 0121$ & 3 & 3.6 & 447 & 0.5 & & & 0.167 & & 4.06 & tail of high-speed stream \\
\hline$* 223$ & $1977 / 10 / 1920$ & 3 & 7.2 & 447 & 0.4 & 0.220 & 0.056 & 0.123 & 0.022 & 1.76 & data gap, high-speed stream? \\
\hline 24 & 1978/09/29 18 & 3 & 18.1 & 705 & 0.5 & 0.051 & 0.003 & 0.387 & 0.131 & 1.22 & associated with a flux rope \\
\hline
\end{tabular}

$* 1, * 2, * 3, * 4$ : a series of events

low-density periods. The thick dashed curves in the temperature panels are the temperatures calculated from solar wind speed using the correlation between solar wind speed and proton temperature (Lopez, 1987). They are plotted to identify abnormally depressed proton temperatures associated with transient events (Richardson and Cane, 1995). The solar wind with abnormally depressed proton temperatures was not detected in the low-density solar wind periods shown in Fig. 7, where the values of plasma beta and $P_{\mathrm{dyn}}$ in lowdensity solar wind were approximately one hundredth of the values in the proceeding solar wind at their minimum values. The thick dashed curves in the plasma beta panels in Fig. 7 shows the Alfven Mach number $\left(M_{A}\right)$. The value of $M_{A}$ was approximately one near the density minima in the low-density regions. The dotted and dashed curves in the total pressure $\left(P_{\text {tot }}\right)$ panels in Fig. 7 shows the gas pressure of solar wind $\left(P_{\text {gas }}\right)$ and the magnetic pressure of solar wind $\left(P_{\text {mag }}\right)$. The $P_{\text {gas }}$ decreased in the low-density solar wind. However, the $P_{\text {tot }}$ in the low-density solar wind kept balance with surrounding solar wind by the $P_{\text {mag }}$. The directions of the interplanetary magnetic fields followed well the spiral of Archimedes and fluctuations of the interplanetary magnetic field decreased in the low-density regions.

The low-density solar winds on 4 and 31 July 1979 were observed in the away IMF sector while the 11-12 May 1999 event preceded a sector boundary. The event on 4 July 1979, recurred on 31 July 1979, suggesting that it was a corotating rather than a transient structure. In the events on 31 July 1979, and 11-12 May 1999, the density decreased gradually, and its recovery time was shorter than the time it took to decrease while the density decrease of the 4 July 1979 event looks symmetrical.

Figure 8 shows synoptic maps of the solar source surface field, from the Wilcox Observatory, associated with the three low-density events given in Fig. 7. Circles in Fig. 8 show the probable source regions of the low-density events. The source regions were determined by mapping back to the Sun using the solar wind speed at $1 \mathrm{AU}$. The suggested source regions of the 4 and 31 July 1979 events were almost in the middle of the large positive polarity region. The source of 


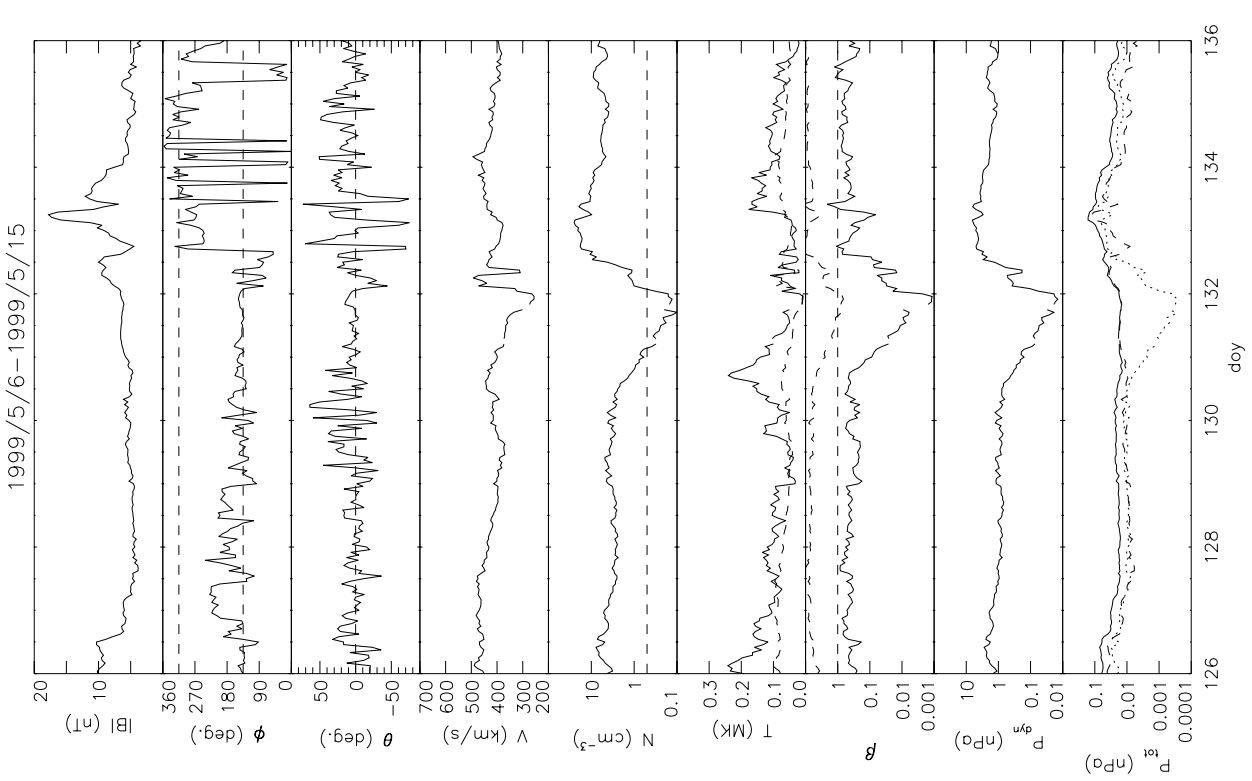

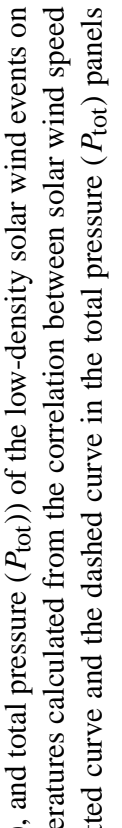

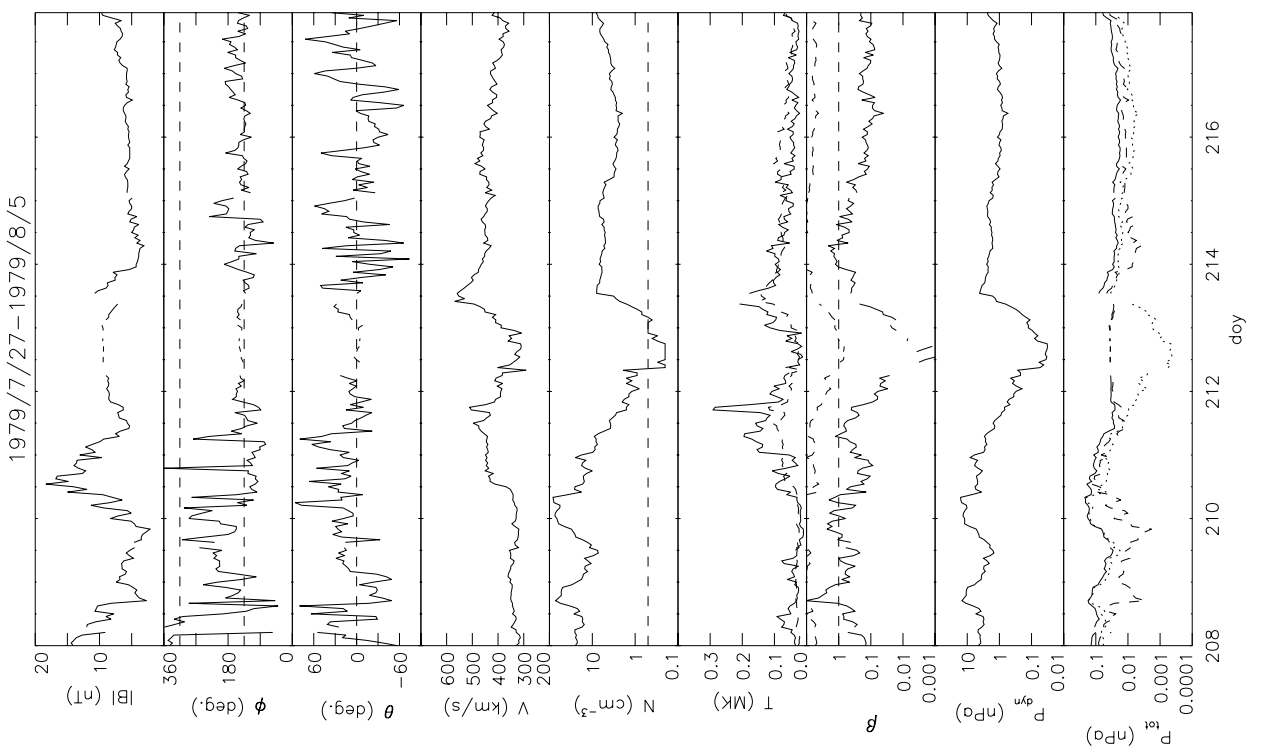

है워 훙

$\underset{0}{0}$

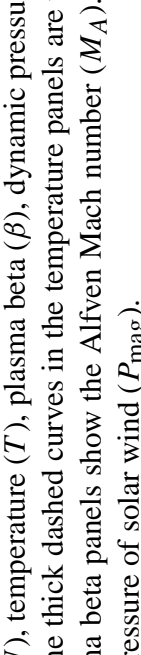

گ芯

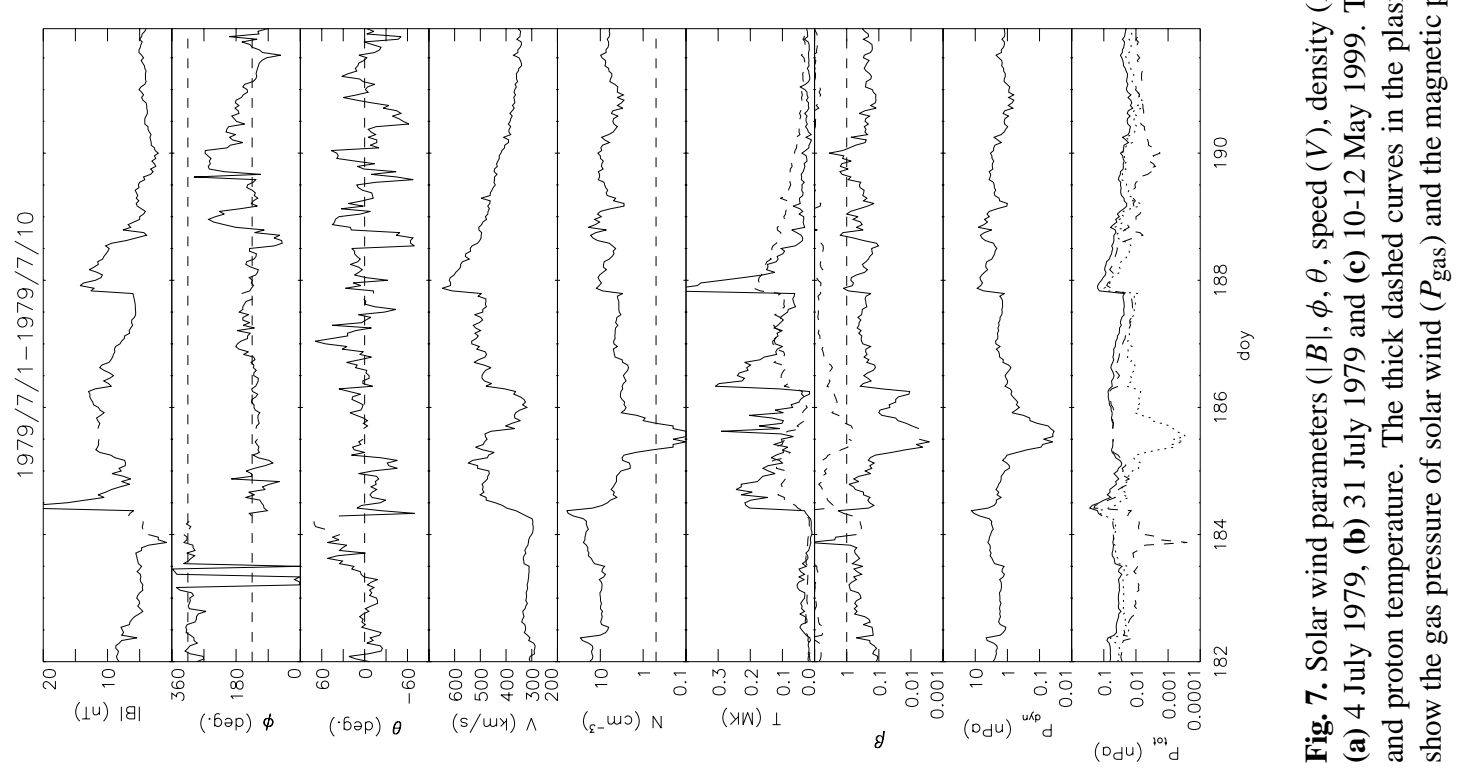




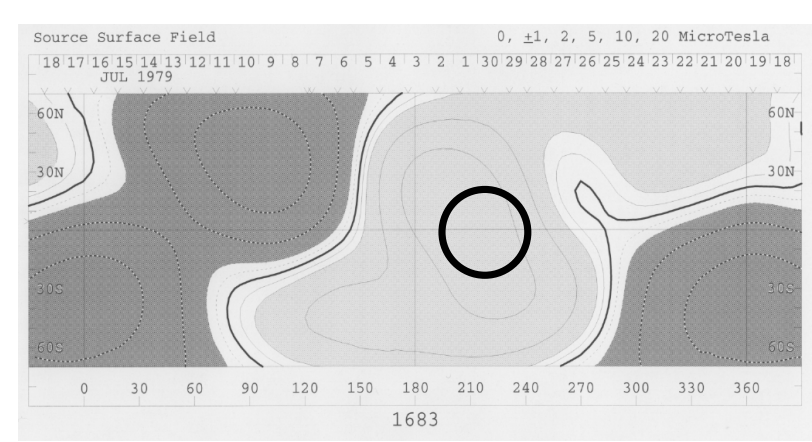

(a) 4 July 1979

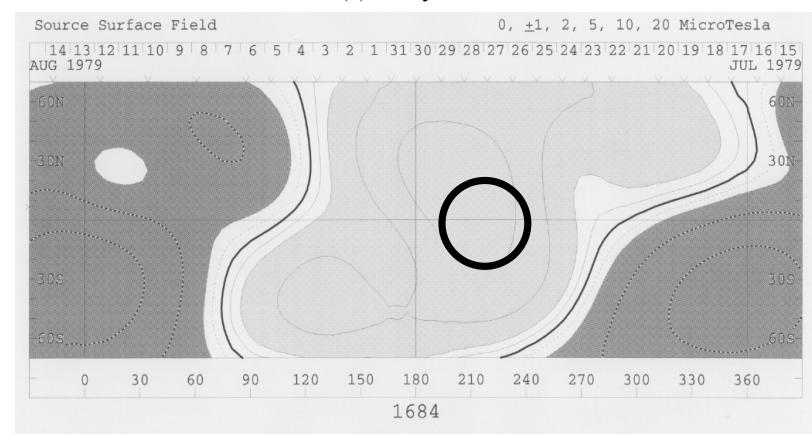

(b) 31 July 1979

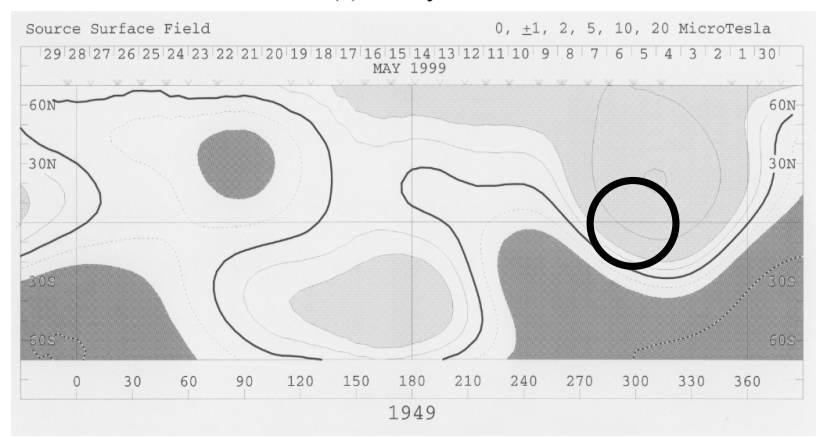

(c) 11-12 May 1999

Fig. 8. Synoptic maps of source surface field made by the Wilcox Observatory. Circles show the probable source regions of three long-duration low-density solar wind events shown in Fig. 7.

the 11-12 May 1999 is suggested to be in the large positive polarity region.

\section{Summary}

We found that the duration of the low-density solar wind event of 11-12 May 1999 was 22 hours, making it the longest event in our database when we selected solar winds with density $0.5 \mathrm{~cm}^{-3}$ or less. Several low-density solar wind events have been observed since 1963. For those low-density events, plasma beta, Alfven Mach number, and dynamic pres- sure were low. In the low-density regions the total pressure kept balance with surrounding solar wind by slightly increased magnetic pressure while the gas pressure decreased significantly. Approximately $20 \%$ of the low-density events were associated with high-speed stream and approximately half of the low-density events with duration three hours or longer were associated with transient events (e.g., flux rope, ejecta, and shock). We found that the low-density events observed on 4 and 31 July 1979 and on 1 and 27 December 1969 showed recurrent activity. The events on 1 and 27 December 1969 were observed in the tail of high-speed streams.

Acknowledgements. We would like to thank the NASA/NSSDC for use of the OMNI database, the ACE Science Center for the use of solar wind data measured by the ACE spacecraft, Dr. D. J. McComas (Los Alamos National Laboratory) for the ACE/SWEPAM data, and Drs. N. F. Ness, L'Heureux, and C. W. Smith (Bartol Research Institute) and Drs. L. Burlaga and M. Acuna (NASA/GSFC) for the ACE/MAG data, the Wilcox Observatory for the synoptic maps of the solar source surfaces.

Topical Editor E. Antonucci thanks E. Cliver and another Referee for their help in evaluating this paper.

\section{References}

Cairns, I. H., Fairfield, D. H., Anderson, R. R., Carlton, V. E. H., Paularena, K. I., and Lazarus, A. J., Unusual location of Earth's bow shock on September 24-25, 1987: Mach number effects, J. Geophys. Res., 100, 47-62, 1995.

Fairfield, D. H., Average and unusual locations of the Earth's magnetopause and bow shock, Ann. Geophysicae, 76, 6700-6716, 1971.

Hundhausen, A. J., The solar wind, in Introduction to space physics, Eds. M. G. Kivelson and C. T. Russell, Cambridge Univ. Press, pp. 91-128, 1995.

Kawano, H., Russell, C. T., and Newbury, J. A., Correlation between the solar wind dynamic and static pressures, J. Geophys. Res., 105, 7583-7589, 2000.

Lopez, R., Solar cycle invariance in solar wind proton temperature relationships, J. Geophys. Res., 92, 11189-11194, 1987.

Newbury, J. A., Russell, C. T., Phillips, J. L., and Gary, S. P., Electron temperature in the ambient solar wind: Typical properties and a lower bound at 1 AU, J. Geophys. Res., 103, 9553-9566, 1998.

Richardson, I. G. and Cane, H. V., Regions of abnormally low proton temperature in the solar wind (1965-1991) and their association with ejecta., J. Geophys. Res., 100, 23397-23412, 1995.

Sibeck, D. G., Lopez, R. E., and Roelof, E. C., Solar wind control of the magnetopause shape, location, and motion, J. Geopys. Res., 96, 5489-5495, 1991.

Takeuchi, T., Araki, T., Lepping, R. P., Yamamoto, T., Kokubun, S., Nagai, T., and Iemori, T., A magnetic cloud with unusual structure and corresponding bow shock movement observed on May 13, 1995, Geophys. Res. Lett., 25, 3269-3272, 1998. 